\title{
Recurrencia de enfermedades glomerulares en trasplante renal
}

\author{
Recurrence of glomerular diseases in kidney transplantation
}

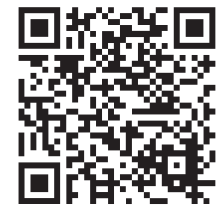

\author{
Francisco Monteón-Ramos, ${ }^{*}$ Luis Alberto Evangelista-Carrillo* \\ * Secretario Técnico del Consejo Estatal de Trasplantes de Órganos y Tejidos (CETOT), Estado de Jalisco. \\ ‡ División de Nefrología y Trasplante. Hospital de Especialidades Centro Médico Nacional de Occidente, IMSS. Guadalajara, Jalisco.
}

\section{CAUSAS DE ENFERMEDAD RENAL TERMINAL (ERCT): RIESGO DE RECIDIVA}

Las glomerulonefritis (GN) primarias continúan siendo una de las principales causas de enfermedad renal terminal en EE.UU. y a nivel mundial. En 2018 el Registro de Diálisis y Trasplante de Australia, Nueva Zelanda (ANZDATA) reportó $17 \%$ de pacientes incidentes con enfermedad renal crónica terminal (ERCT) en quienes se documentó una GN. En EE.UU. 7 y $13 \%$ de pacientes incidentes en diálisis o que recibieron un trasplante renal (TR), respectivamente, tenían GN. ${ }^{1-5}$ En México no hay reportes sobre la incidencia de GN en pacientes que inician diálisis o reciben un TR, pero más de $90 \%$ de pacientes reciben este tratamiento sin saber la causa de la ERCT (Tabla 1). ${ }^{6}$

Posterior a TR las GN de novo o recurrentes son una importante causa de falla prematura del injerto. Todas las variedades histológicas primarias o secundarias pueden recurrir de 3 a 15\%, especialmente las de alto riesgo. En México la experiencia es limitada y la recurrencia se reporta de $4.6 \%$ en 1,321 biopsias de injertos renales en el Hospital de Especialidades del Centro Médico Nacional de Occidente del IMSS, ${ }^{6}$ por lo tanto se desconoce la pérdida de injertos por las diferentes variedades histológicas y no hay manejo sistematizado para estas lesiones.

Las limitaciones que se tienen para sistematizar su diagnóstico son un problema mundial, más aún en México (Tabla 2). Destacan el desconocimiento de la causa de la ERCT en la mayoría de los casos, la diversidad en la indicación de biopsia renal en el injerto, y la carencia de recursos técnicos para su diagnóstico. Asimismo, destaca la carencia de microscopia con inmunofluorescencia y electrónica en la mayoría de los centros de trasplantes a nivel nacional así como de patólogos renales, entre otras muchas limitaciones, incluso relacionadas con la precisión para el diagnóstico. ${ }^{2}$

La correlación clínica, patológica y laboratorial ayudará a precisar posibles factores que influyen en el riesgo de recurrencia, por lo que el clínico necesita una historia clínica precisa para tratar de elucidar la temporalidad de la lesión renal en los riñones nativos y la progresión del daño renal, causas de pérdidas de injertos previos, etc. ${ }^{2}$ Además, la disponibilidad de recursos de laboratorio especializado es esencial para un diagnóstico exacto, así por ejemplo en las lesiones de alto riesgo de recurrencia como la glomeruloesclerosis focal y segmentaria (GSFyS), la medición del factor de permeabilidad circulante y más recientemente del receptor activador de plasminógeno de tipo uroquinasa soluble (suPAR) en sangre y orina, respectivamente, ayudan a detectar y manejar de manera adecuada casos con alto riesgo de recurrencia (ver Tabla 3). En la GN membranosa el autoanticuerpo circulante frente al receptor tipo $M$ de la fosfolipasa $A 2$ (anti-PLA2R) se ha convertido en un marcador pronóstico y se han correlacionado niveles de este marcador con respuesta a inmunosupresores o incluso a remisión espontánea. 
Se recomienda que pacientes con esta lesión deben ser trasplantados con niveles bajos y así evitar el riesgo de recurrencia, aunque aún no hay estudios clínicos controlados que marquen la pauta pronóstica en esta lesión. La medición del anti-PLA2R se encuentra disponible en varias ciudades del país y es de extrema utilidad en esta lesión glomerular. ${ }^{2,7}$

Los marcadores de recurrencia como los anticuerpos dirigidos contra la membrana basal glomerular, medición de cadenas ligeras kappa y lambda y la medición de ADAMTS13 y potenciales mutaciones del factor regulador del complemento ya es posible medirlos para el diagnóstico y tratamiento de las lesiones glomerulares secundarias y por lo tanto, un mejor manejo, reducirá su recurrencia en caso de necesitar trasplante renal (Tabla 3). ${ }^{8}$

La Tabla 4 nos indica las posibles opciones de tratamiento recomendadas como manejo inicial así como alternativas en caso de no respuesta o respuesta parcial e incluso información sobre indicaciones basadas en estudios clínicos controlados. Estas opciones son para glomerulopatías de alto riesgo de recurrencia y son de extrema utilidad clínica para el manejo de estos pacientes. Cabe destacar que la mayoría de ellos se encuentran disponibles en México, por lo que se resalta la trascendencia de tener un diagnóstico histopatológico preciso para tratar de obtener los mejores resultados y evitar el daño estructural por la recurrencia de la enfermedad glomerular de los riñones nativos.

Todo lo anterior nos lleva a afirmar que las enfermedades glomerulares, incluso de alto riesgo, no son contraindicación para trasplante renal con una buena evaluación clínica, diagnóstico histológico correcto así como las medidas de prevención y el recurso diagnóstico y terapéutico adecuado, que en muchos centros de trasplante renal a nivel nacional están disponibles. Por lo tanto, las enfermedades sistémicas no son contraindicación para trasplante renal, siempre y cuando se encuentren en remisión cuando menos 3-6 meses previos a la cirugía. En casos, por ejemplo, dos trasplantes con recurrencia de GSFyS en el injerto debe evitarse donación familiar o buscar donadores con muerte cerebral y establecer manejo de prevención como plasmaféresis, rituximab y más

Tabla 1: Prevalencia, riesgo de pérdida de injerto y factores predictores de recurrencia de glomerulonefritis postrasplante renal. ${ }^{5}$

\begin{tabular}{|c|c|c|c|c|c|}
\hline Registro & Todos & Nefropatía por lgA & $\begin{array}{l}\text { Glomeruloesclerosis } \\
\text { focal y segmentaria }\end{array}$ & Nefropatía membranosa & Membranoproliferativa \\
\hline \multicolumn{6}{|c|}{ Prevalencia de recurrencia } \\
\hline ANZDATA (1985-2014) & $10.3 \%$ & $\begin{array}{l}10 \% \text { a } 10 \text { años, } \\
15 \% \text { a } 15 \text { años }\end{array}$ & $\begin{array}{l}9 \% \text { a } 10 \text { años, } \\
11 \% \text { a } 15 \text { años }\end{array}$ & $\begin{array}{l}16 \% \text { a } 10 \text { años, } \\
18 \% \text { a } 15 \text { años }\end{array}$ & $\begin{array}{l}16 \% \text { a } 10 \text { años, } \\
19 \% \text { a } 15 \text { años }\end{array}$ \\
\hline Mayo/Toronto & $39.5 \%$ a 5 años & $\begin{array}{l}42 \% \text { a } 3 \text { años, } \\
51 \% \text { a } 5 \text { años }\end{array}$ & $\begin{array}{l}31 \% \text { a } 3 \text { años, } \\
35 \% \text { a } 5 \text { años }\end{array}$ & $\begin{array}{l}45 \% \text { a } 3 \text { años, } \\
55 \% \text { a } 5 \text { años }\end{array}$ & $\begin{array}{l}41 \% \text { a } 3 \text { años, } \\
41 \% \text { a } 5 \text { años }\end{array}$ \\
\hline $\begin{array}{l}\text { British Columbia } \\
(1990-2005)\end{array}$ & $\begin{array}{l}13 \% \text { a } 10 \text { años, } \\
18 \% \text { a } 15 \text { años }\end{array}$ & $15.4 \%$ & $9.7 \%$ & $10 \%$ & $4.8 \%$ (GNMP tipo 1) \\
\hline Corea $(1995-2010)$ & $17.8 \%$ & $14.8 \%$ & $6.3 \%$ & $0 \%$ & $12.5 \%$ \\
\hline Francia & No reportado & $36 \%$ a 10 años & No reportado & No reportado & No reportado \\
\hline \multicolumn{6}{|c|}{ Pérdida de injerto (5 años posterior a recurrencia) } \\
\hline ANZDATA (1985-2014) & $55 \%$ & $58 \%$ & $57 \%$ & $59 \%$ & $30 \%$ \\
\hline $\begin{array}{l}\text { Registro de enferme- } \\
\text { dades de injerto renal } \\
(1987-1996)\end{array}$ & $40 \%$ & $41 \%$ & $65 \%$ & $44 \%$ & $66 \%$ \\
\hline Mayo/Toronto & $H R: 2.6(1.9-3.6)$ & HR: $3.4(1.2-9.7)$ & HR: $5(2.4-10-1)$ & HR: $1.4(0.3-6.8)$ & HR: $6.8(2.7-17.2)$ \\
\hline British Columbia & HR: $7.5(5.5-10.2)$ & No reportado & No reportado & No reportado & No reportado \\
\hline Corea (1995-2010) & HR: 4 (1.7-9.3) & No reportado & No reportado & No reportado & No reportado \\
\hline $\begin{array}{l}\text { Predictores clínicos de } \\
\text { recurrencia }\end{array}$ & $\begin{array}{c}\text { Enfermedad renal } \\
\text { secundaria a glomerulo- } \\
\text { nefritis primaria, género } \\
\text { masculino, jóvenes, no } \\
\text { blancos, sin esteroides }\end{array}$ & $\begin{array}{l}\text { Jóvenes, libres de } \\
\text { esteroides, no utilizar } \\
\text { inducción (se sugiere } \\
\text { timoglobulina) }\end{array}$ & $\begin{array}{l}\text { Jóvenes, rápida progre- } \\
\text { sión de la enfermedad } \\
\text { renal }\end{array}$ & $\begin{array}{l}\text { Presencia y títulos de } \\
\text { anti-PRA2R pretras- } \\
\text { plante }\end{array}$ & $\begin{array}{c}\text { Glomerulopatía por C3, } \\
\text { presencia de gammapa } \\
\text { tía monoclonal, escasa } \\
\text { respuesta al tratamiento } \\
\text { y rápida progresión de } \\
\text { riñones nativos }\end{array}$ \\
\hline
\end{tabular}

GNMP = glomerulonefritis membranoproliferativa; $\mathrm{HR}$ = hazard ratio (razón de las tasas de riesgo).

Modificado de: Lim WH, Shingde M, Wong G. Recurrent and de novo glomerulonephritis after kidney transplantation. Front Immunol. 2019; 10: 1944. doi: 10.3389/fimmu.2019.01944. ${ }^{5}$ 
Tabla 2: Limitaciones para el diagnóstico de las glomerulonefritis recurrentes. ${ }^{1}$

Enfermedad original en riñones nativos

No conocida en la mayoría de los pacientes con IRC

No conocida cuando se presenta en etapa tardía

Dificultad para diferenciar lesiones primarias o secundarias como en GSFyS

Enfermedad renal en donador

Glomerulonefritis transmitida y glomerulopatía

Indicaciones para efectuar biopsia renal del injerto

Falta de indicación uniforme para biopsia en injerto con/sin disfunción Proteinuria y hematuria con disfunción renal

Biopsia de protocolo vs. por indicación

Diagnóstico en glomerulonefritis postrasplante

Carencia en hallazgos histológicos en GSFyS en etapa temprana de recurrencia

Dificultad para diferenciar GNMP vs. glomerulopatía del trasplante

Dificultad para diferenciar nefropatía por IgA primaria vs. secundaria

Dificultad para diferenciar la causa de síndrome urémico hemolítico/púrpura

trombocitopénica trombótica

Primario vs. toxicidad a drogas vs. rechazo humoral

IRC = insuficiencia renal crónica; GSFyS = glomeruloesclerosis focal y segmentaria; GNMP = glomerulonefritis membranoproliferativa; trombocitopénica trombótica.

recientemente abatacept y otros compuestos, con los cuales con hay experiencia extensa en su uso, y sólo son casos anecdóticos. Pautas similares se establecen en los trasplantes con GN membranosa donde gracias al nivel de anti-PLA2R, al uso de rituximab, e incluso bortezomib, se han tenido casos con buena respuesta. ${ }^{5,9}$

En este contexto el manejo postrasplante renal es esencial. La disfunción renal, proteinuria incluso menor de 300 mg en 24 horas y hematuria macro o microscópica en dos o más determinaciones con sonograma renal en límites, sin obstrucción y resto de estudios de laboratorio sin datos anormales deben ser indicación de biopsia renal y en caso de biopsia protocolizada, la detección de lesiones glomerulares recurrentes es de mayor utilidad clínica y terapéutica, pues nos permite un tratamiento más temprano.

Finalmente, se sumaría esta información a las guías de manejo de las lesiones glomerulares más recurrentes después de trasplante renal.

\section{Guía de recurrencia en Glomeruloesclerosis} Focal y Segmentaria ${ }^{10}$

Evaluación laboratorial: mutaciones de podocinas, NPHS28 (GSFyS familiar) o estudio genético en niños y adultos y suPAR (no validado en estudios controlados) (GSFyS no familiar). Estudios no disponibles en México de momento.
Profilaxis: datos sugieren que proteína sérica (factor circulante correlaciona con recurrencia). Hay datos inconsistentes y no fácilmente disponibles en México. No hay información científica de calidad del uso de la plasmaféresis previo al trasplante renal ni reportes no controlados sobre resultados positivos posteriores a trasplante renal. El uso de rituximab en caso de recurrencia permanece incierto.

\section{Sugerencias:}

a) Candidatos a trasplante renal con GSFyS deben ser advertidos del riesgo de recurrencia y pérdida del injerto.

b) GSFyS no contraindica el trasplante.

c) Historia previa de GSFyS con pérdida de injerto se considera una contraindicación relativa para donación viva.

d) Ensayos de laboratorio para determinar factor circulante no son validados y no están disponibles en práctica clínica, suPAR prometedor en vías de validación.

e) Estudios nuevos muestran que la combinación de terapia con plasmaféresis y rituximab induce remisión parcial o total en $57 \%$ de los casos.

\section{Guía de recurrencia en nefropatía por $\operatorname{Ig} \mathrm{A}^{10}$}

Evaluación laboratorial: deficiencia de galactosa de la IgA I y el CD89 soluble pueden estar implicados en la patogénesis, progresión y posible recurrencia. Estudios no disponibles en México.

Tabla 3: Posibles factores que influyen en la recurrencia de las glomerulonefritis.

Glomeruloesclerosis focal y segmentaria $1,5,8$

Edad de inicio

Intervalo hasta la falla renal terminal

Historia de pérdida de injerto por glomeruloesclerosis focal y segmentaria

Factores de permeabilidad circulantes

Factor circulante de uroquinasa

Glomerulonefritis membranosa

Concentración de anticuerpo contra el receptor de antifosfolipasa A2 (anti-PLA2R)

Glomerulonefritis por anticuerpos antimembrana basal glomerular

Titulación de anticuerpos anti-membrana basal glomerular

Enfermedad por depósito de cadenas ligeras

Amiloidosis AL

Glomerulopatía paraproteinémicas

Detección de cadenas ligeras libres, relación kappa/lambda

Síndrome urémico hemolítico atípico

Actividad disminuida de ADMTS13

Mutación del factor regulador del complemento I, $\mathrm{H}$

Algunas glomerulonefritis membranoproliferativas tipo II (enfermedad de depósi-

tos densos)

Mutación del factor regulador del complemento I, H, C3 NeF 
Monteón-Ramos F y col. Recurrencia de enfermedades glomerulares en trasplante renal

Tabla 4: Opciones de tratamiento propuestas para recurrencia de enfermedades glomerulares postrasplante. ${ }^{5}$

\begin{tabular}{llll}
\hline & \multicolumn{1}{c}{ Tratamiento inicial } & \multicolumn{1}{c}{ Otras opciones } & Ensayos clínicos en proceso \\
\hline Nefropatía por IgA & $\begin{array}{l}\text { Anti-proteinúricos } \\
\text { Inhibidor de calcineurina más }\end{array}$ & $\begin{array}{l}\text { Agentes alquilantes } \\
\text { Amigdalectomía }\end{array}$ & Inducción (basiliximab vs. timoglobulina) \\
& esteroides & Ofatumumab & Pre-emptive rituximab \\
Glomeruloesclerosis focal & Anti-proteinúricos & Abatacept/belatacept & Bleselumab \\
y segmentaria & Recambio plasmático & Radiación linfoide total \\
Refropatía membranosa & Rituximab & Rituximab (anti-PLA2R negativo) & \\
& Anti-proteinúricos & Bortezomib & \\
Inhibidor de calcineurina & Agentes alquilantes & Eculizumab (nefropatía por C3) \\
Membranoproliferativa & Rituximab (anti-PLA2R positivo) & Recambio plasmático e inmunoab- & \\
& Anti-proteinúricos & sorción & Agentes alquilantes o rituximab si es \\
\hline
\end{tabular}

Modificado de: Lim WH, Shingde M, Wong G. Recurrent and de novo glomerulonephritis after kidney transplantation. Front Immunol. 2019; 10: 1944. doi: 10.3389/fimmu.2019.01944.5

Profilaxis: ninguna. Sugerencias:

a) Los pacientes deben ser informados acerca del alto riesgo de recurrencia, pero con curso benigno en la mayoría de los casos.

b) La recurrencia puede ser más común en receptores jóvenes, donante vivo full match y lesión rápidamente progresiva.

c) La recurrencia de nefropatía por IgA no es una contraindicación para retrasplante.

d) Los agentes alquilantes (en caso de medias lunas) y la tonsilectomía son opciones que no se usan sistemáticamente.

Guía de recurrencia para glomerulonefritis membranoproliferativa (GNMP) ${ }^{10}$

Evaluación laboratorial: determinación de complemento, C3 Neff (glomerulopatía por C3) factor $\mathrm{H} \mathrm{y}$ medición de $\lg G$ monoclonales especialmente $\lg G 3$ kappa, IgG 3 lambda, IgG 2 lambda, la mayoría no disponibles en México. Se recomienda en GNMP por complejos inmunes determinar anticuerpo para hepatitis C (forma secundaria).

Profilaxis: el uso de plasmaféresis, ciclofosfamida y rituximab permanece controversial. Eculizumab ha mostrado resultados prometedores en la glomerulopatía por C3.

\section{Sugerencias:}

a) Todos los pacientes con GNMP, ya sea mediada por complejos inmunes o por complemento, deben ser informados de la enfermedad original y pérdida del injerto.

b) El diagnóstico de GNMP no contraindica el trasplante renal.

Guía de recurrencia para glomerulonefritis membranosa $(\mathrm{GNM})^{10}$

Evaluación laboratorial: la determinación del antiPLA2R se usa como marcador diagnóstico y pronóstico en esta lesión glomerular y en la recurrencia de la enfermedad en el trasplante renal. Hay reportes de casos clínicos bien documentados. Prueba de laboratorio en la cual los niveles de este marcador pueden definir riesgos de recurrencia. Prueba disponible en México. Otro marcador, el autoantígeno tipo trombospondina tipo I que contiene el dominio 7A ha mostrado resultados prometedores.

Profilaxis: ninguna.

\section{Sugerencias:}

a) Todo paciente con GNM debe ser informado del riesgo de recurrencia y pérdida del injerto.

b) El diagnóstico de GNM no contraindica el trasplante renal.

\section{Guía de recurrencia para amiloidosis ${ }^{10}$}

Evaluación laboratorial: ninguna en amiloidosis primaria y secundaria.

Profilaxis: ninguna. Se recomienda uso de colchicina en la forma secundaria por fiebre familiar mediterránea. 


\section{Sugerencias:}

a) Los pacientes no deben ser trasplantados en caso de involucro sistémico, ya sea severo, sobre todo cardiaco, y de alta mortalidad.

b) Los pacientes en muchas ocasiones se encuentran debilitados.

c) Los reportes sobre trasplante combinado de riñón y medula ósea parecen prometedores.

\section{REFERENCIAS}

1. Morozumi K, Takeda A, Otsuka Y, Horike K, Gotoh N, Watarai $Y$. Recurrent glomerular disease after kidney transplantation: an update of selected areas and the impact of protocol biopsy. Nephrology (Carlton). 2014; 19 Suppl 3: 6-10.

2. Choy BY, Chan TM, Lai KN. Recurrent glomerulonephritis after kidney transplantation. Am J Transplant. 2006; 6 (11): 25352542.

3. Ponticelli C, Moroni G, Glassock RJ. De novo glomerular diseases after renal transplantation. Clin J Am Soc Nephrol. 2014; 9 (8): 1479-1487.

4. Canaud G, Audard V, Kofman T, Lang P, Legendre C, Grimbert $P$. Recurrence from primary and secondary glomerulopathy after renal transplant. Transpl Int. 2012; 25 (8): 812-824.

5. Lim WH, Shingde M, Wong G. Recurrent and de novo glomerulonephritis After Kidney Transplantation. Front Immunol. 2019; 10: 1944.
6. Pazarin Villaseñor L, Villanueva-Pérez A, Ramos Solano F, Monteon Ramos F, Rojas Campos E. Prevalencia de glomerulopatias posterior al trasplante renal en el occidente de México [Resumen]. LVII Reunión Anual IMIN 2008.

7. Dabade TS, Grande JP, Norby SM, Fervenza FC, Cosio FG. Recurrent idiopathic membranous nephropathy after kidney transplantation: a surveillance biopsy study. Am J Transplant. 2008; 8 (6): 1318-1322.

8. Ponticelli C, Moroni G, Glassock RJ. Recurrence of secondary glomerular disease after renal transplantation. Clin J Am Soc Nephrol. 2011; 6 (5): 1214-1221.

9. Uffing A, Pérez-Sáez MJ, Mazzali M, Manfro RC, Bauer AC, de Sottomaior Drumond F et al. Recurrence of FSGS after Kidney Transplantation in Adults. Clin J Am Soc Nephrol. 2020; 15 (2): 247-256.

10. Kasiske BL, Ramos EL, Gaston RS, Bia MJ, Danovitch GM, Bowen PA et al. The evaluation of renal transplant candidates: clinical practice guidelines. Patient Care and Education Committee of the American Society of Transplant Physicians. J Am Soc Nephrol. 1995; 6 (1): 1-34.

Correspondencia:

Dr. Francisco Monteón-Ramos

E-mail: francisco.monteon@jalisco.gob.mx 